\title{
Review: smoking is associated with decreased bone mineral density and increased risk of hip fracture in postmenopausal women
}

\author{
Law MR, Hackshaw AK. A meta-analysis of cigarette smoking, bone mineral density and risk of hip fracture: recognition of a \\ major effect. BMJ 1997 Oct 4;315:841-6.
}

\section{Question}

Does smoking lead to decreased bone mineral density and increased risk of hip fracture in premenopausal and postmenopausal women?

\section{Data sources}

Studies were identified from a Medline search using the terms smoking or tobacco combined with the terms bone density, osteoporosis, or hip fracture. References from relevant papers were also reviewed.

\section{Study selection}

Studies were selected if they assessed bone mineral density or hip fracture in women according to smoking habit. Studies of patients with specific diseases or those who started smoking later in life were excluded.

\section{Data extraction}

Data were extracted on age adjusted bone mineral density in the femoral neck, radius, or calcaneus; the difference between the mean bone mineral density of women who smoked and those who did not smoke; incidence of hip fracture; age adjusted relative risk (RR) of hip fracture based on smoking habit; and menopausal status.

\section{Main results}

29 cross sectional studies reported bone mineral density in 2156 women who smoked and 9705 women who did not smoke. 19 cohort and case control studies reported 3889 hip fractures in primarily postmenopausal women. The average number of cigarettes smoked daily was about 15 . In postmenopausal women, bone mineral density was lower in women who smoked compared with those who did not smoke, and the difference increased with age $(p=0.001)$. For each 10 year increase in age, the bone mineral density of women who smoked fell below that of women who did not smoke by 0.14 SD (95\% CI 0.07 to 0.21 SD; approximately $2 \%$, CI $1 \%$ to $3 \%$ ) relative to the average bone mineral density at the onset of menopause. By age 80 , the bone mineral density of women who smoked was $0.45 \mathrm{SD}$ (CI 0.34 to $0.56 \mathrm{SD}$; approximately $6 \%$ ) lower than that of women who did not smoke. The association of smoking with bone mineral density was similar for all bone sites. In premenopausal women, bone mineral density was similar in smoking and non-smoking groups.

RR of hip fracture was higher for women who smoked compared with women who did not smoke and the risk increased with age $(p<0.001)$. Smoking had no effect on risk of hip fracture in premenopausal women; but increased risk at age 60 years (RR 1.17, CI 1.05 to 1.30 ), age 70 years (RR 1.41, CI 1.29 to 1.55 ), age 80 years (RR 1.71 , CI 1.50 to 1.96 ), and age 90 years (RR 2.08, CI 1.70 to 2.54). Among all women, 13\% of hip fractures were attributable to smoking.

RR of hip fracture was indirectly estimated using bone mineral density differences. Similar estimates of the risk of hip fracture at different ages in women who smoked were derived directly from studies of smoking and hip fracture and indirectly from studies of smoking and bone mineral density.

\section{Conclusions}

Postmenopausal women who smoke have lower bone mineral density and an increased risk of hip fracture compared with women who do not smoke. These differences increase with age. Smoking is not associated with lower bone mineral density or increased hip fracture in premenopausal women.

Source of funding: no external funding.

For article reprint: not available.

A modified version of this abstract appears in ACP Journal Club 1998 Mar-Apr.

\section{Commentary}

The meta-analysis by Law and Hackshaw expands our knowledge about the relation between cigarette smoking, bone mineral density, and risk of hip fracture. Previous research has recognised that smoking is a major cause of hip fracture in postmenopausal women. In 1994, the National Osteoporosis Foundation confirmed this in their study of 300000 patients with osteoporosis related hip fractures. ${ }^{1}$ Law and Hackshaw conclude that smoking increases the lifetime risk of hip fracture by about half, from an estimated $12 \%$ to $19 \%$ in women up to age 85 , and from $22 \%$ to $37 \%$ to age $90.13 \%$ of all hip fractures are attributable to smoking.

Law and Hackshaw found similar results when the risk of hip fracture was derived directly from studies of smoking and hip fracture and indirectly from studies of smoking and bone density. This consistency in findings further strengthens the causal argument. It would have been helpful for readers if the 19 cohort and case control studies and the 29 cross sectional studies had been tabulated, clearly stating the purpose, method, sampling, and findings of each study. Also, analysis of differences and similarities among the selected studies, an important consideration when interpreting the results, would have been valuable.

From a clinical standpoint, this research is beneficial in confirming the importance of advising patients with osteoporosis to quit smoking. Because smoking from the time of menopause onwards increases by half the risk of hip fracture in old age, and because stopping smoking prevents further excess bone loss, advising women to stop smoking at the time of menopause should eliminate the excess risk

$$
\begin{array}{r}
\text { Kyung Rim Shin, RN, EdD } \\
\text { Associate Professor } \\
\text { College of Nursing Science } \\
\text { Ewha Women's University } \\
\text { Seoul, Korea }
\end{array}
$$

1 Melton LJ 3rd, Thamer M, Ray NF, et al. Fractures attributable to osteoporosis: report Fractures attributable to osteoporosis: report
from the National Osteoporosis Foundation. $J$ Bome Miner Res 1997;12:16-23. 